\title{
New Constructions of Asymptotically Optimal Codebooks via Cyclotomic Classes of Order 8
}

\author{
Wanfeng Qi $\left(\mathbb{D},{ }^{1}\right.$ Yueying Song, ${ }^{1}$ Rui Ma, ${ }^{1}$ Lingli Tang $\left(\mathbb{D},{ }^{2}\right.$ and Qian Wang ${ }^{1}$ \\ ${ }^{1}$ School of Mathematics, Liaoning Normal University, Dalian, Liaoning 116024, China \\ ${ }^{2}$ College of Science, Dalian Minzu University, Dalian, Liaoning 116600, China \\ Correspondence should be addressed to Lingli Tang; tanglingli@dlnu.edu.cn
}

Received 31 December 2018; Accepted 20 February 2019; Published 10 March 2019

Academic Editor: Luigi Rodino

Copyright (c) 2019 Wanfeng Qi et al. This is an open access article distributed under the Creative Commons Attribution License, which permits unrestricted use, distribution, and reproduction in any medium, provided the original work is properly cited.

\begin{abstract}
Asymptotically optimal codebooks are a family of codebooks that can approach an optimal codebook meeting the Welch bound when the lengths of codewords are large enough. They can be constructed easily and are a good alternative for optimal codebooks in many applications. In this paper, we construct a new class of asymptotically optimal codebooks by using the product of some special finite fields and almost difference sets, which are composed of cyclotomic classes of order eight.
\end{abstract}

\section{Introduction}

An $(N, K)$ codebook $\mathscr{C}$ is a set $\left\{c_{0}, c_{1}, \ldots, c_{N-1}\right\}$, where each codeword $c_{l}, 0 \leq l \leq N-1$, is a unit norm complex vector in $\mathbb{C}^{K}$. The value of maximum cross-correlation amplitude of an $(N, K)$ codebook $\mathscr{C}$ is defined by

$$
I_{\text {max }}(\mathscr{C})=\max _{0 \leq i<j \leq N-1}\left|c_{i} c_{j}^{H}\right|,
$$

where $c^{H}$ denotes the conjugate transpose of a complex vector c. Codebooks with minimal possible $I_{\max }(\mathscr{C})$ are desirable in many applications. From a geometric point of view, the minimal possible $I_{\max }(\mathscr{C})$ means that the smallest angle between two different lines where the codewords are located is as large as possible. Welch [1] presented the following wellknown lower bound for $I_{\max }(\mathscr{C})$.

Lemma 1 (see [1]). For any $(N, K)$ codebook $C$ with $N \geq K$,

$$
I_{\max }(\mathscr{C}) \geq I_{\text {Welch }}=\sqrt{\frac{N-K}{(N-1) K}} .
$$

Moreover, the equality holds if and only if, for all pairs of $(i, j)$ with $i \neq j$, it holds that

$$
\left|c_{i} c_{j}^{H}\right|=\sqrt{\frac{N-K}{(N-1) K}} .
$$

A codebook $\mathscr{C}$ meeting the Welch bound is called a maximum-Welch-bound-equality (MWBE) codebook. The interests in MWBE codebooks have significantly increased because of their special properties. However, it is usually difficult to construct a family of MWBE codebooks $[2,3]$.

Most existing methods for constructing infinite families MWBE codebooks fall into three categories: strongly regular graph methods [4], difference sets methods [5], and Steiner systems methods [6].

It is known that the Welch bound is not tight when $N>$ $K^{2}$. In fact, $N<K^{2}$ is only the necessary condition of the existence of an $(N, K)$ MWBE codebook [2]. Even when $N<K^{2}$ holds, the corresponding $(N, K)$ MWBE codebook may not exist [7]. In recent years, asymptotically optimal codebooks have attracted widespread attention, since they can be constructed easily and $I_{\max }(\mathscr{C})$ can approach the Welch bound for large enough $N$. Thus, they are good alternatives in many applications.

Many methods can be used to construct asymptotically optimal codebooks, such as almost difference sets [8-10], binary sequences [11], and character sums in finite fields [12, 13]. Hu and $\mathrm{Wu}$ [14] constructed some asymptotically optimal codebooks by using difference sets and the product of Abelian groups. In this paper, we generalize this method to the case of cyclotomic classes of order 8 in finite fields and propose new classes of codebooks asymptotically meeting the Welch bound. 
This paper is organized as follows. In Section 2, we recall some basic theory and definitions that will be used in our discussion. In Section 3, we present our main results. In Section 4, we give conclusions and future work.

\section{Preliminaries}

Let $p$ be a prime number; $q=p^{n}, n \geq 1$. For a finite field $\mathbb{F}_{q}$, let $\theta$ be its one fixed primitive element, and let $\mathbb{F}_{q}^{*}$ be its all nonzero elements.

Let $q=e f+1, e \geq 2, f \geq 2$. The $\operatorname{set} C_{j}^{(e, q)}:=\left\{\theta^{e k+j}, k \in\right.$ $\mathbb{Z}\}=\left\{\theta^{e k+j}, k=0,1, \ldots, f-1\right\}, j=0,1, \ldots, e-1$ is called a cyclotomic class of order $e$. Clearly, $\mathbb{F}_{q}^{*}=\bigcup_{j=0}^{e-1} C_{j}^{(e, q)}$, and $\left|C_{j}^{(e, q)}\right|=f, j=0,1, \ldots, e-1$.

The cyclotomic numbers of order $e$ with respect to $\mathbb{F}_{q}$ are given by

$$
(l, m)_{e}=\left|\left(C_{l}^{(e, q)}+1\right) \cap C_{m}^{(e, q)}\right|,
$$

where $S+a:=\{s+a: s \in S\}$. Cyclotomic number $(l, m)_{e}$ is the number of solutions to the equation $1=y-x, x \in$ $C_{l}^{(e, q)}, y \in C_{m}^{(e, q)}$.

Cyclotomic class is now widely used to construct almost difference sets. An almost difference set $D$ of a finite field $\mathbb{F}_{q}$ is a subset with $k$ elements satisfying special properties. Define

$$
\triangle(x)=\left\{\left(d, d^{\prime}\right) \in D^{2}: d-d^{\prime}=x\right\} .
$$

$D$ is called a $(q, k, \lambda, t)$ almost difference set of $\mathbb{F}_{q}$ if $|\triangle(x)|=\lambda$ for $t$ nonzero elements of $\mathbb{F}_{q}$, and $|\triangle(x)|=\lambda+1$ for the remaining $q-1-t$ nonzero elements of $\mathbb{F}_{q}$. When $q \equiv$ $1(\bmod 4), C_{0}^{(2, q)}$ is a $(q,(q-1) / 2,(q-5) / 4,(q-1) / 2)$ almost difference set, which is also called the Paley partial difference set [15].

Let $q=8 f+1$; Lehmer [16] presented the explicit form of the 64 cyclotomic numbers of order $e=8$ when $q \equiv 9(\bmod 16)$, which are functions whose parameters are determined by the unique representation

$$
\begin{aligned}
& q=x^{2}+4 y^{2}=a^{2}+2 b^{2} \\
& x, y, a, b \in \mathbb{Z}, x \equiv a \equiv 1(\bmod 4) .
\end{aligned}
$$

The 64 cyclotomic numbers have two forms determined by whether 2 is a quartic residue in $\mathbb{F}_{q}$ or not. Let $D=C_{0}^{(8, q)} \cup$ $C_{1}^{(8, q)} \cup C_{2}^{(8, q)} \cup C_{5}^{(8, q)}, \triangle_{j}=\left|\left(D+\theta^{j}\right) \cap D\right|, 0 \leq j \leq 7$. When 2 is a quartic residue in $\mathbb{F}_{q}$, Ding et al. [17] calculated that

$$
\begin{aligned}
& \triangle_{0}=\triangle_{4}=\frac{16 q-48+8 x-8 a-48 y}{64}, \\
& \triangle_{1}=\triangle_{5}=\frac{16 q-80-16 x+16 a-32 y}{64}, \\
& \triangle_{2}=\triangle_{6}=\frac{16 q-48+8 x-8 a-16 y}{64}, \\
& \triangle_{3}=\triangle_{7}=\frac{16 q-16}{64} .
\end{aligned}
$$

When 2 is not a quartic residue in $\mathbb{F}_{q}$, Zhang and Feng [10] calculated that

$$
\begin{aligned}
& \triangle_{0}=\triangle_{4}=\frac{16 q-48+8 x-8 a+16 y}{64}, \\
& \triangle_{1}=\triangle_{5}=\frac{16 q-80}{64}, \\
& \triangle_{2}=\triangle_{6}=\frac{16 q-48+8 x-8 a-16 y}{64}, \\
& \triangle_{3}=\triangle_{7}=\frac{16 q-16-16 x+16 a}{64} .
\end{aligned}
$$

If $l$ is a power of a prime and $l=t^{2}+2 \equiv 3(\bmod 8)$, then, for $q=l^{2}$, Ding [17] proved that $q \equiv 9(\bmod 16), x=-l$, $y=0, a=-l+4, b= \pm 2 t$, and 2 is a quartic residue in $F_{q}^{*}$, and $D=C_{0}^{(8, q)} \cup C_{1}^{(8, q)} \cup C_{2}^{(8, q)} \cup C_{5}^{(8, q)}$ is a $(q,(q-1) / 2,(q-5) / 4,(q-$ 1)/2) almost difference set of $\mathbb{F}_{q}$. Thus, $C_{k}^{(8, q)} \cup C_{1+k}^{(8, q)} \cup C_{2+k}^{(8, q)} \cup$ $C_{5+k}^{(8, q)}, \quad k=0,1, \ldots, 7$ are also $(q,(q-1) / 2,(q-5) / 4,(q-1) / 2)$ almost difference sets of $\mathbb{F}_{q}$.

An additive character of $\mathbb{F}_{q}$ with characteristic $p$ is a homomorphism from $\mathbb{F}_{q}$ to the unit circle in the complex plane, which in fact has the following form [18]:

$$
\chi_{c}(g)=\exp \left(\frac{2 \pi \sqrt{-1} \operatorname{Tr}(c g)}{p}\right), \quad \forall g \in \mathbb{F}_{q}, c \in \mathbb{F}_{q},
$$

where $\operatorname{Tr}$ is the trace function from $\mathbb{F}_{q}$ to $\mathbb{F}_{p}$. Multiplicative characters of $\mathbb{F}_{q}$ are characters of the multiplicative group $\mathbb{F}_{q}^{*}$. Let $\theta$ be a fixed primitive element of $\mathbb{F}_{q}$. All multiplicative characters $\psi_{j}$ of $\mathbb{F}_{q}$ have the following form [18]:

$$
\psi_{j}\left(\theta^{k}\right)=\exp \left(\frac{2 \pi \sqrt{-1} j k}{q-1}\right)
$$

$$
\text { for } k=0,1, \ldots, q-2, j=0,1, \ldots, q-2 \text {. }
$$

The set of all additive characters of $\mathbb{F}_{q}$ is denoted by $\widehat{\mathbb{F}}_{q}$, and $\left|\widehat{\mathbb{F}}_{q}\right|=q$. When $c=0, \chi_{0}$ is trivial, which satisfies $\chi_{0}(g)=$ $1, \forall g \in \mathbb{F}_{q}$. For a subset $D$ and an additive character $\chi$ of $\mathbb{F}_{q}, \chi(D)$ is defined to be $\chi(D)=\sum_{g \in D} \chi(g)$. Clearly, $\chi(\bar{D})=$ $-\chi(D)$.

For $m$ additive characters $\chi_{1}, \chi_{2}, \ldots, \chi_{m}$ of $\mathbb{F}_{q}$, we can define their product as

$$
\left(\chi_{1} \chi_{2} \ldots \chi_{m}\right)(g)=\chi_{1}(g) \chi_{2}(g) \ldots \chi_{m}(g)
$$

for any $g \in \mathbb{F}_{q}$.

The direct product of $n$ finite fields $\mathbb{F}_{q_{1}}, \mathbb{F}_{q_{2}}, \ldots, \mathbb{F}_{q_{n}}$ can be defined by

$$
\begin{aligned}
\mathbb{F}_{q_{1}} & \times \mathbb{F}_{q_{2}} \times \cdots \times \mathbb{F}_{q_{n}} \\
& =\left\{\left(g_{1}, g_{2}, \ldots, g_{n}\right), g_{i} \in \mathbb{F}_{q_{i}}, i=1, \ldots, n\right\} .
\end{aligned}
$$

Correspondingly, an additive character $\chi_{1} \otimes \chi_{2} \otimes \cdots \otimes \chi_{n}$ on the direct product is defined by

$$
\begin{gathered}
\left(\chi_{1} \otimes \chi_{2} \otimes \cdots \otimes \chi_{n}\right)\left(g_{1}, g_{2}, \ldots, g_{n}\right) \\
=\chi_{1}\left(g_{1}\right) \chi_{2}\left(g_{2}\right) \cdots \chi_{n}\left(g_{n}\right),
\end{gathered}
$$


where $\left(g_{1}, g_{2}, \ldots, g_{n}\right) \in \mathbb{F}_{q_{1}} \times \mathbb{F}_{q_{2}} \times \cdots \times \mathbb{F}_{q_{n}}$ and $\chi_{1} \in \widehat{\mathbb{F}}_{q_{1}}, \chi_{2} \in$ $\widehat{\mathbb{F}}_{q_{2}}, \ldots, \chi_{n} \in \widehat{\mathbb{F}}_{q_{n}}$.

\section{Main Result}

In this section, we will follow Hu's method [14] to construct asymptotically optimal codebooks. Hu's method [14] used the product of difference sets of finite Abelian groups of order $N_{i}$, where $N_{i} \equiv 3(\bmod 4)$. In our paper, we use two classes of $(q,(q-1) / 2,(q-5) / 4,(q-1) / 2)$ almost difference sets in finite fields: one class is $C_{k}^{(8, q)} \cup C_{1+k}^{(8, q)} \cup C_{2+k}^{(8, q)} \cup C_{5+k}^{(8, q)}, k=$ $0,1, \ldots, 7, q \equiv 9(\bmod 16)$, and the other is $C_{0}^{(2, q)}$ and $C_{1}^{(2, q)}$, $q \equiv 1(\bmod 4)$.

For the case of $D=C_{0}^{(8, q)} \cup C_{1}^{(8, q)} \cup C_{2}^{(8, q)} \cup C_{5}^{(8, q)}$, we first estimate $\chi(D)$ for any nontrivial additive characters of a finite field. In fact, Zhang and Feng had given an upper bound of $\chi(D)$ in Theorem 1 of [10]. We rephrase it in our setting.

Lemma 2. Let $\mathbb{F}_{q}$ be a finite field, $q \equiv 9(\bmod 16)$, and $x, y, a, b$ are determined by (6). $D=C_{0}^{(8, q)} \cup C_{1}^{(8, q)} \cup$ $C_{2}^{(8, q)} \cup C_{5}^{(8, q)} \cdot \chi(\cdot)=\chi_{c}(\cdot)=\exp (2 \pi \sqrt{-1} \operatorname{Tr}(c \cdot) / p)$ is a nontrivial additive character. If $\lim _{q->\infty}|x-a| / \sqrt{q}=0$ and $\lim _{q->\infty}|y| / \sqrt{q}=0$, then there exists a function $f(q)$ satisfying $\lim _{q \rightarrow \infty} f(q) / q=0$ such that

$$
\begin{aligned}
& |\chi(D)| \leq \frac{\sqrt{q+f(q)}}{2}, \\
& |\chi(\bar{D})| \leq \frac{\sqrt{q+f(q)}}{2} .
\end{aligned}
$$

Proof. Let $\eta_{j}=\sum_{g \in C_{j}^{(8, q)}} \exp (2 \pi \sqrt{-1} \operatorname{Tr}(g) / p) . \eta_{j}$ is a Gauss period of order 8 . Since $c \neq 0$, let $c \in C_{\lambda}^{(8, q)}$. Then

$$
\begin{aligned}
|\chi(D)|^{2} & =\sum_{x \in D} \sum_{y \in D} \exp \left(\frac{2 \pi \sqrt{-1} \operatorname{Tr}(c(x-y))}{p}\right) \\
& =\frac{q-1}{2} \\
& +\sum_{x \in D} \sum_{y \neq x, y \in D} \exp \left(\frac{2 \pi \sqrt{-1} \operatorname{Tr}(c(x-y))}{p}\right) \\
& =\frac{q-1}{2} \\
& +\sum_{j=0}^{7} \sum_{x \in D} \sum_{y \in D, x-y \in C_{j}^{(8, q)}} \exp \left(\frac{2 \pi \sqrt{-1} \operatorname{Tr}(c(x-y))}{p}\right) \\
& =\frac{q-1}{2}+\sum_{j=0}^{7} \eta_{j+\lambda} \triangle_{j}=\frac{q-1}{2}
\end{aligned}
$$

$$
\begin{aligned}
& +\sum_{j=0}^{7} \eta_{j+\lambda}\left(\triangle_{j}-\frac{q-1}{4}+\frac{q-1}{4}\right)=\frac{q-1}{4} \\
& +\sum_{j=0}^{7} \eta_{j+\lambda}\left(\triangle_{j}-\frac{q-1}{4}\right) .
\end{aligned}
$$

Note that, whether 2 is a quartic residue in $\mathbb{F}_{q}$ or not, $\triangle_{i}=$ $\triangle_{i+2}, i=0,1,2,3$. Thus

$$
|\chi(D)|^{2}=\frac{q-1}{4}+\sum_{j=0}^{3}\left(\eta_{j+\lambda}+\eta_{j+4+\lambda}\right)\left(\triangle_{j}-\frac{q-1}{4}\right) .
$$

Let $\psi$ be the multiplicative character satisfying $\psi(\theta)=\psi_{(q-1) / 4}\left(\theta^{1}\right)=\exp (2 \pi \sqrt{-1} / 4) \cdot \psi\left(g \theta^{-j}\right)=$ $\psi(g) \exp (-2 \pi \sqrt{-1} j / 4)$. So if $g \in C_{j}^{(8, q)} \cup C_{j+4}^{(8, q)}$, we have $\sum_{i=0}^{3} \psi^{i}\left(g \theta^{-j}\right)=4$, or else $\sum_{i=0}^{3} \psi^{i}\left(g \theta^{-j}\right)=0$. Thus,

$$
\begin{aligned}
\eta_{j}+\eta_{j+4} & =\sum_{g \in C_{j}^{(8, q)} \cup C_{j+4}^{(8, q)}} \exp \left(\frac{2 \pi \sqrt{-1} \operatorname{Tr}(g)}{p}\right) \\
& =\frac{1}{4} \sum_{g \in \mathbb{F}_{q}^{*}} \exp \left(\frac{2 \pi \sqrt{-1} \operatorname{Tr}(g)}{p}\right) \sum_{i=0}^{3} \psi^{i}\left(g \theta^{-j}\right) \\
& =\frac{1}{4} \sum_{i=0}^{3} G\left(\psi^{i}\right) \exp \left(\frac{-2 \pi \sqrt{-1} i j}{4}\right),
\end{aligned}
$$

where $G(\psi)=\sum_{g \in \mathbb{F}_{q}^{*}} \psi(g) \exp (2 \pi \sqrt{-1} \operatorname{Tr}(g) / p)$ is the Gaussian sum over $\mathbb{F}_{q}$ and $G\left(\psi^{0}\right)=-1,\left|G\left(\psi^{i}\right)\right|=\sqrt{q}, i=1,2,3$ [18]. Thus, $\left|\eta_{j}+\eta_{j+4}\right| \leq(1 / 4)(1+3 \sqrt{q})$.

Then

$$
\begin{aligned}
|\chi(D)|^{2} & =\frac{q-1}{4}+\sum_{j=0}^{3}\left(\eta_{j+\lambda}+\eta_{j+4+\lambda}\right)\left(\triangle_{j}-\frac{q-1}{4}\right) \\
& \leq \frac{q-1}{4}+\frac{1}{4}(1+3 \sqrt{q}) \sum_{j=0}^{3}\left|\triangle_{j}-\frac{q-1}{4}\right| .
\end{aligned}
$$

When 2 is a quartic residue in $\mathbb{F}_{q}$, substituting (7) into (18) yields

$$
|\chi(D)|^{2} \leq \frac{q+1}{4}+\frac{3 \sqrt{q}}{2}+\frac{1+3 \sqrt{q}}{8}(|x-a|+3|y|) .
$$

When 2 is a quartic nonresidue in $\mathbb{F}_{q}$, substituting (8) into (18) yields

$$
|\chi(D)|^{2} \leq \frac{q+1}{4}+\frac{3 \sqrt{q}}{2}+\frac{1+3 \sqrt{q}}{8}(|x-a|+|y|) .
$$

Denote $f(q)=1 / 4+3 \sqrt{q} / 2+((1+3 \sqrt{q}) / 8)(|x-a|+3|y|)$. If $\lim _{q->\infty}|x-a| / \sqrt{q}=0$ and $\lim _{q->\infty}|y| / \sqrt{q}=0$, then $\lim _{q->\infty} f(q) / q=0$. Thus

$$
|\chi(D)| \leq \frac{\sqrt{q+f(q)}}{2} .
$$


Since $\chi(\bar{D})=-\chi(D)$,

$$
|\chi(\bar{D})|=|\chi(D)| \leq \frac{\sqrt{q+f(q)}}{2} .
$$

Remark 3. There exist infinitely many $x, y, a, b$ satisfying the assumptions in Lemma 2. Ding et al. [17] proved that if $q=$ $l^{2} \equiv 9(\bmod 16), l=t^{2}+2 \equiv 3(\bmod 8)$, then $x=$ $-l, y=0, a=-l+4, b= \pm 2 t$, where $t$ is an integer. In this case, we have $|x-a|=4,|y|=0, D$ becomes a $(q,(q-1) / 2,(q-5) / 4,(q-1) / 2)$ almost difference set, and $|\chi(D)| \leq \sqrt{q+12 \sqrt{q}+3} / 2$.

Remark 4. Lemma 2 still holds if we use $\widetilde{D}=C_{l}^{(8, q)} \cup C_{1+l}^{(8, q)} \cup$ $C_{2+l}^{(8, q)} \cup C_{5+l}^{(8, q)}, l=1, \ldots, 7$ instead of $D=C_{0}^{(8, q)} \cup C_{1}^{(8, q)} \cup C_{2}^{(8, q)} \cup$ $C_{5}^{(8, q)}$. The reason is that new $\widetilde{\triangle}_{j}=\left|\left(\widetilde{D}+\theta^{j}\right) \cap \widetilde{D}\right|$ is equal to $\triangle_{j-l}$. Thus, it has no influence on estimating $|\chi(D)|$. However, if we use other combinations of four cyclotomic classes which do not correspond to a family of almost difference sets, there may not exist infinitely many $x, y, a, b$ satisfying the assumptions in Lemma 2 . The reason is that the eight $\widetilde{\triangle}_{j}, j=$ $0, \ldots, 7$ will have more different values, which inevitably lead to more restrictions on $x, y, a, b$. Thus, it is more difficult to find infinitely many $q$ such that $|\chi(D)|$ is not too large.

Remark 5. Since $D=C_{0}^{(8, q)} \cup C_{2}^{(8, q)} \cup C_{4}^{(8, q)} \cup C_{6}^{(8, q)}$ can be seen as $C_{0}^{(2, q)}$, we consider $C_{0}^{(2, q)}$ and $C_{1}^{(2, q)}$, where $q \equiv 1(\bmod 4)$. Ding [19] proved that $\chi\left(C_{0}^{(2, q)}\right)=(-1 \pm \sqrt{q}) / 2$ and $\chi\left(C_{1}^{(2, q)}\right)=$ $(-1 \mp \sqrt{q}) / 2$. Thus, for $D=C_{0}^{(2, q)}$ or $D=C_{1}^{(2, q)}$, we have $\max |\chi(D)|=(\sqrt{q}+1) / 2$.

Suppose that $\mathbb{F}_{q_{1}}, \mathbb{F}_{q_{2}}, \ldots, \mathbb{F}_{q_{n}}$ are $n$ finite fields. Let $\mathbb{F}=$ $\mathbb{F}_{q_{1}} \times \mathbb{F}_{q_{2}} \times \ldots \times \mathbb{F}_{q_{n}}$. Then $\hat{\mathbb{F}}=\left\{\chi_{1, j_{1}} \otimes \chi_{2, j_{2}} \otimes \cdots \otimes \chi_{n, j_{n}} \mid\right.$ $\left.\chi_{i, j_{i}} \in \widehat{\mathbb{F}}_{q_{i}}, 1 \leq i \leq n\right\}$.

Let $D_{i}=C_{0}^{\left(8, q_{i}\right)} \cup C_{1}^{\left(8, q_{i}\right)} \cup C_{2}^{\left(8, q_{i}\right)} \cup C_{5}^{\left(8, q_{i}\right)}$ be the union of the four cyclotomic classes of order 8 in $\mathbb{F}_{q_{i}}, q_{i} \equiv 9(\bmod 16), i=$ $1, \ldots, n$. Let $\overline{D_{i}}$ be the complement of $D_{i}$ with respect to $\mathbb{F}_{q_{i}}$. Note that $\left|D_{i}\right|=\left(q_{i}-1\right) / 2$.

Let $E_{1}=D_{1}$. For any $1 \leq i \leq n-1$, put

$$
E_{i+1}=\left(E_{i} \times \bar{D}_{i+1}\right) \cup\left(\bar{E}_{i} \times D_{i+1}\right) .
$$

Finally, let $D=E_{n}$, and $K=|D|$. Let $N=|\mathbb{F}|$; then $N=$ $q_{1} q_{2} \cdots q_{n}$.

We can define similar sets in the case of $\mathbb{F}_{q_{i}}, q_{i} \equiv$ $1(\bmod 4), i=1, \ldots, n$. More specifically, let $D_{i}=C_{0}^{\left(2, q_{i}\right)} \mathrm{C}$ $\mathbb{F}_{q_{i}}, q_{i} \equiv 1(\bmod 4), i=1, \ldots, n$. Then we can define $\bar{D}_{i}$, $E_{i}, i=1, \ldots, n$ in a similar way.

For both of the two cases, we have the following lemma.

Lemma 6. $\left|E_{i}\right|=\left(q_{1} q_{2} \cdots q_{i}-1\right) / 2$ for any $1 \leq i \leq n$. In particular, $K=(N-1) / 2$.
Proof. For $i=1$, we have $\left|E_{1}\right|=\left(q_{1}-1\right) / 2$. For $i>1$, suppose that $\left|E_{i}\right|=\left(q_{1} q_{2} \ldots q_{i}-1\right) / 2$. Then, it follows that

$$
\begin{aligned}
\left|E_{i+1}\right|= & \frac{q_{1} q_{2} \cdots q_{i}-1}{2} \cdot \frac{q_{i+1}+1}{2}+\frac{q_{1} q_{2} \cdots q_{i}+1}{2} \\
& \cdot \frac{q_{i+1}-1}{2}=\frac{\left(q_{1} q_{2} \cdots q_{i+1}-1\right)}{2} .
\end{aligned}
$$

Therefore, $\left|E_{n}\right|=\left(q_{1} q_{2} \cdots q_{n}-1\right) / 2=(N-1) / 2$.

We define an $(N, K)$ codebook by

$$
\begin{aligned}
& \mathscr{C}_{D} \\
& \quad=\left\{C_{j_{1}, j_{2}, \ldots, j_{n}} \mid j_{i}=0,1, \ldots, q_{i}-1, i=1,2, \ldots, n\right\} .
\end{aligned}
$$

The vector $C_{j_{1}, j_{2}, \ldots, j_{n}} \in C^{K}$ is defined by

$$
\begin{aligned}
& C_{j_{1}, j_{2}, \ldots, j_{n}}=\frac{1}{\sqrt{K}}\left(\chi_{1, j_{1}} \otimes \chi_{2, j_{2}} \otimes \cdots\right. \\
& \left.\left.\quad \otimes \chi_{n, j_{n}}\right)\left(g_{i, 1}, g_{i, 2}, \ldots, g_{i, n}\right)\right)_{i=1}^{K} \\
& \quad=\frac{1}{\sqrt{K}}\left(\chi_{1, j_{1}}\left(g_{i, 1}\right) \chi_{2, j_{2}}\left(g_{i, 2}\right) \ldots \chi_{n, j_{n}}\left(g_{i, n}\right)\right)_{i=1}^{K},
\end{aligned}
$$

where $\chi_{i, j_{i}} \in \widehat{\mathbb{F}}_{q_{i}},\left(g_{i, 1}, g_{i, 2}, \ldots, g_{i, n}\right) \in D$ and $\chi_{i, 0}$ is the corresponding trivial character. It is obvious that $\left|\mathscr{C}_{D}\right|=N$, and the length of the vector $C_{j_{1}, j_{2}, \ldots, j_{n}}$ is $|D|=K$.

Next we will investigate $I_{\max }\left(\mathscr{C}_{D}\right)$. For the case of $D_{i}=$ $C_{0}^{\left(8, q_{i}\right)} \cup C_{1}^{\left(8, q_{i}\right)} \cup C_{2}^{\left(8, q_{i}\right)} \cup C_{5}^{\left(8, q_{i}\right)} \subset \mathbb{F}_{q_{i}}, q_{i} \equiv 9(\bmod 16)$, we need the following lemma.

Lemma 7. With the above notations, for any character $\chi_{1, j_{1}} \otimes$ $\chi_{2, j_{2}} \otimes \cdots \otimes \chi_{n, j_{n}}$ of $\mathbb{F}_{q}, j_{i}=0,1, \ldots, q_{i}-1, q_{i} \equiv 9(\bmod 16), i=$ $1,2, \ldots, n$. If $\left(j_{1}, j_{2}, \ldots, j_{n}\right)=(0,0, \ldots, 0)$, we have

$$
\left|\left(\chi_{1, j_{1}} \otimes \chi_{2, j_{2}} \otimes \cdots \otimes \chi_{n, j_{n}}\right)(D)\right|=K .
$$

If $\left(j_{1}, j_{2}, \ldots, j_{n}\right) \neq(0,0, \ldots, 0)$,

$$
\begin{aligned}
& \left|\left(\chi_{1, j_{1}} \otimes \chi_{2, j_{2}} \otimes \cdots \otimes \chi_{n, j_{n}}\right)(D)\right| \\
& \quad \leq \frac{\sqrt{q_{1}+f\left(q_{1}\right)} \cdots \sqrt{q_{n}+f\left(q_{n}\right)}}{2} .
\end{aligned}
$$

Proof. If $n=1$, when $j_{1}=0, \chi_{1, j_{1}}$ is the trivial character; thus, $\chi_{1, j_{1}}(D)=|D|=\left(q_{1}-1\right) / 2=K$. When $j_{1} \neq 0$, $\chi_{1, j_{1}}$ is a nontrivial character. Thus, by Lemma $2,\left|\chi_{1, j_{1}}(D)\right| \leq$ $\sqrt{q_{1}+f\left(q_{1}\right)} / 2$.

If $n=2$, we need to consider three cases:

(1) $j_{1}=j_{2}=0$

$$
\left|\left(\chi_{1, j_{1}} \otimes \chi_{2, j_{2}}\right)(D)\right|=|D|=\left|E_{2}\right|=\frac{q_{1} q_{2}-1}{2}=K .
$$


(2) $j_{1}=0, j_{2} \neq 0$

$$
\begin{aligned}
\left(\chi_{1, j_{1}} \otimes \chi_{2, j_{2}}\right)(D)= & \chi_{1, j_{1}}\left(D_{1}\right) \chi_{2, j_{2}}\left(\bar{D}_{2}\right) \\
& +\chi_{1, j_{1}}\left(\bar{D}_{1}\right) \chi_{2, j_{2}}\left(D_{2}\right) \\
= & \left|D_{1}\right| \chi_{2, j_{2}}\left(\bar{D}_{2}\right)+\left|\bar{D}_{1}\right| \chi_{2, j_{2}}\left(D_{2}\right) \\
= & \left(\left|D_{1}\right|-\left|\bar{D}_{1}\right|\right) \chi_{2, j_{2}}\left(\bar{D}_{2}\right) \\
& +\left|\bar{D}_{1}\right| \chi_{2, j_{2}}\left(\mathbb{F}_{q_{2}}\right) .
\end{aligned}
$$

Thus,

$$
\begin{aligned}
\left|\left(\chi_{1, j_{1}} \otimes \chi_{2, j_{2}}\right)(D)\right| & =\left|\left(\left|D_{1}\right|-\left|\bar{D}_{1}\right|\right) \chi_{2, j_{2}}\left(\bar{D}_{2}\right)\right| \\
& \leq|| D_{1}|-| \bar{D}_{1}|| \frac{\sqrt{q_{2}+f\left(q_{2}\right)}}{2} \\
& =\frac{\sqrt{q_{2}+f\left(q_{2}\right)}}{2} \\
& <\frac{\sqrt{q_{1}+f\left(q_{1}\right)} \sqrt{q_{2}+f\left(q_{2}\right)}}{2} .
\end{aligned}
$$

In a similar way, if $j_{1} \neq 0, j_{2}=0$,

$$
\begin{aligned}
\left|\left(\chi_{1, j_{1}} \otimes \chi_{2, j_{2}}\right)(D)\right| & \leq \frac{\sqrt{q_{1}+f\left(q_{1}\right)}}{2} \\
& <\frac{\sqrt{q_{1}+f\left(q_{1}\right)} \sqrt{q_{2}+f\left(q_{2}\right)}}{2} .
\end{aligned}
$$

(3) $j_{1} \neq 0, j_{2} \neq 0$

$$
\begin{aligned}
& \left|\left(\chi_{1, j_{1}} \otimes \chi_{2, j_{2}}\right)(D)\right| \\
& \quad=\left|\chi_{1, j_{1}}\left(D_{1}\right) \chi_{2, j_{2}}\left(\bar{D}_{2}\right)+\chi_{1, j_{1}}\left(\bar{D}_{1}\right) \chi_{2, j_{2}}\left(D_{2}\right)\right| \\
& \quad=2\left|\chi_{1, j_{1}}\left(D_{1}\right) \chi_{2, j_{2}}\left(D_{2}\right)\right| \\
& \quad \leq \frac{\sqrt{q_{1}+f\left(q_{1}\right)} \cdot \sqrt{q_{2}+f\left(q_{2}\right)}}{2} .
\end{aligned}
$$

Now we assume that the formula holds for $n-1$; we will prove it for $n$.

If $\left(j_{1}, j_{2}, \ldots, j_{n}\right)=(0,0, \ldots, 0)$, then the character $\chi_{1, j_{1}} \otimes$ $\chi_{2, j_{2}} \otimes \cdots \otimes \chi_{n, j_{n}}$ is trivial. Hence, the result is correct. In the following, we consider the case of $n>2$ and $\left(j_{1}, j_{2}, \ldots, j_{n}\right) \neq$ $(0,0, \ldots, 0)$. The proof can also be divided into three cases:
Case $(1)\left(\left(j_{1}, j_{2}, \ldots, j_{n-1}\right)=(0,0, \ldots, 0), j_{n} \neq 0\right)$. We compute

$$
\begin{aligned}
& \left(\chi_{1, j_{1}} \otimes \chi_{2, j_{2}} \otimes \cdots \otimes \chi_{n, j_{n}}\right)(D) \\
& \quad=\left(\chi_{1, j_{1}} \otimes \chi_{2, j_{2}} \otimes \cdots \otimes \chi_{n, j_{n}}\right) \\
& \quad \cdot\left(\left(E_{n-1} \times \bar{D}_{n}\right) \cup\left(\bar{E}_{n-1} \times D_{n}\right)\right)=\left|E_{n-1}\right| \chi_{n, j_{n}}\left(\bar{D}_{n}\right) \\
& \quad+\left|\bar{E}_{n-1}\right| \chi_{n \cdot j_{n}}\left(D_{n}\right)=-\left|E_{n-1}\right| \chi_{n \cdot j_{n}}\left(D_{n}\right)+\left|\bar{E}_{n-1}\right| \\
& \quad \cdot \chi_{n \cdot j_{n}}\left(D_{n}\right) .
\end{aligned}
$$

By Lemma 6, $\left|\bar{E}_{n-1}\right|-\left|E_{n-1}\right|=1$. So we have

$$
\begin{aligned}
& \left|\left(\chi_{1, j_{1}} \otimes \chi_{2, j_{2}} \otimes \cdots \otimes \chi_{n, j_{n}}\right)(D)\right|=\left|\chi_{n, j_{n}}\left(D_{n}\right)\right| \\
& \quad \leq \frac{\sqrt{q_{n}+f\left(q_{n}\right)}}{2}<\frac{\sqrt{q_{1}+f\left(q_{1}\right)} \cdots \sqrt{q_{n}+f\left(q_{n}\right)}}{2} .
\end{aligned}
$$

Case $(2)\left(\left(j_{1}, j_{2}, \ldots, j_{n-1}\right) \neq(0,0, \ldots, 0), j_{n}=0\right)$. In this case, we get

$$
\begin{aligned}
& \left(\chi_{1, j_{1}} \otimes \chi_{2, j_{2}} \otimes \cdots \otimes \chi_{n, j_{n}}\right)(D) \\
& \quad=\left(\chi_{1, j_{1}} \otimes \chi_{2, j_{2}} \otimes \cdots \otimes \chi_{n, j_{n}}\right) \\
& \quad \cdot\left(\left(E_{n-1} \times \bar{D}_{n}\right) \cup\left(\bar{E}_{n-1} \times D_{n}\right)\right) \\
& \quad=\left(\chi_{1, j_{1}} \otimes \chi_{2, j_{2}} \otimes \cdots \otimes \chi_{n-1, j_{n-1}}\right)\left(E_{n-1}\right)\left|\bar{D}_{n}\right| \\
& \quad+\left(\chi_{1, j_{1}} \otimes \chi_{2, j_{2}} \otimes \cdots \otimes \chi_{n-1, j_{n-1}}\right)\left(\bar{E}_{n-1}\right)\left|D_{n}\right| \\
& \quad=\left(\chi_{1, j_{1}} \otimes \chi_{2, j_{2}} \otimes \cdots \otimes \chi_{n-1, j_{n-1}}\right)\left(E_{n-1}\right)\left|\bar{D}_{n}\right| \\
& \quad-\left(\chi_{1, j_{1}} \otimes \chi_{2, j_{2}} \otimes \cdots \otimes \chi_{n-1, j_{n-1}}\right)\left(E_{n-1}\right)\left|D_{n}\right| \\
& \quad=\left(\chi_{1, j_{1}} \otimes \chi_{2, j_{2}} \otimes \cdots \otimes \chi_{n-1, j_{n-1}}\right)\left(E_{n-1}\right) .
\end{aligned}
$$

Hence, by the assumption,

$$
\begin{aligned}
& \left|\left(\chi_{1, j_{1}} \otimes \chi_{2, j_{2}} \otimes \cdots \otimes \chi_{n, j_{n}}\right)(D)\right| \\
& \quad=\left|\left(\chi_{1, j_{1}} \otimes \chi_{2, j_{2}} \otimes \cdots \otimes \chi_{n-1, j_{n-1}}\right)\left(E_{n-1}\right)\right| \\
& \quad \leq \frac{\sqrt{q_{1}+f\left(q_{1}\right)} \cdots \sqrt{q_{n-1}+f\left(q_{n-1}\right)}}{2} \\
& \quad<\frac{\sqrt{q_{1}+f\left(q_{1}\right)} \cdots \sqrt{q_{n}+f\left(q_{n}\right)}}{2} .
\end{aligned}
$$

Case $(3)\left(\left(j_{1}, j_{2}, \ldots, j_{n-1}\right) \neq(0,0, \ldots, 0), j_{n} \neq 0\right)$. In this case, we get

$$
\begin{array}{r}
\left(\chi_{1, j_{1}} \otimes \chi_{2, j_{2}} \otimes \cdots \otimes \chi_{n, j_{n}}\right)(D) \\
=\left(\chi_{1, j_{1}} \otimes \chi_{2, j_{2}} \otimes \cdots \otimes \chi_{n, j_{n}}\right)
\end{array}
$$




$$
\begin{aligned}
& \cdot\left(\left(E_{n-1} \times \bar{D}_{n}\right) \cup\left(\bar{E}_{n-1} \times D_{n}\right)\right) \\
& =\left(\chi_{1, j_{1}} \otimes \chi_{2, j_{2}} \otimes \cdots \otimes \chi_{n-1, j_{n-1}}\right)\left(E_{n-1}\right) \cdot \chi_{n, j_{n}}\left(\bar{D}_{n}\right) \\
& +\left(\chi_{1, j_{1}} \otimes \chi_{2, j_{2}} \otimes \cdots \otimes \chi_{n-1, j_{n-1}}\right)\left(\bar{E}_{n-1}\right) \cdot \chi_{n, j_{n}}\left(D_{n}\right) \\
& =-2\left(\chi_{1, j_{1}} \otimes \chi_{2, j_{2}} \otimes \cdots \otimes \chi_{n-1, j_{n-1}}\right)\left(E_{n-1}\right) \\
& \cdot \chi_{n, j_{n}}\left(D_{n}\right) .
\end{aligned}
$$

Hence, by the assumption and Lemma 2,

$$
\begin{aligned}
& \left|\left(\chi_{1, j_{1}} \otimes \chi_{2, j_{2}} \otimes \cdots \otimes \chi_{n, j_{n}}\right)(D)\right| \\
& =\mid-2\left(\chi_{1, j_{1}} \otimes \chi_{2, j_{2}} \otimes \cdots \otimes \chi_{n-1, j_{n-1}}\right)\left(E_{n-1}\right) \\
& \quad \cdot \chi_{n, j_{n}}\left(D_{n}\right) \mid \leq \frac{\sqrt{q_{1}+f\left(q_{1}\right)} \cdots \sqrt{q_{n}+f\left(q_{n}\right)}}{2} .
\end{aligned}
$$

The proof is finished.

Theorem 8. Let $\mathscr{C}_{D}$ be a codebook defined by (25) in the case of $D_{i}=C_{0}^{\left(8, q_{i}\right)} \cup C_{1}^{\left(8, q_{i}\right)} \cup C_{2}^{\left(8, q_{i}\right)} \cup C_{5}^{\left(8, q_{i}\right)} \subset \mathbb{F}_{q_{i}}, q_{i} \equiv 9(\bmod 16)$, and $q_{i}$ satisfies the assumptions in Lemma 2. Then $\mathscr{C}_{D}$ is an $(N, K)=\left(q_{1} q_{2} \cdots q_{n},\left(q_{1} q_{2} \cdots q_{n}-1\right) / 2\right)$ codebook with

$$
I_{\max }\left(\mathscr{C}_{D}\right) \leq \frac{\sqrt{q_{1}+f\left(q_{1}\right)} \cdots \sqrt{q_{n}+f\left(q_{n}\right)}}{q_{1} \cdots q_{n}-1},
$$

and $I_{\max }\left(\mathscr{C}_{D}\right)$ can reach the Welch bound if $q_{i} \longrightarrow \infty$ for any $1 \leq i \leq n$.

Proof. Let $C_{j_{1}, j_{2}, \ldots, j_{n}}$ and $C_{j_{1}^{\prime}, j_{2}^{\prime}, \ldots, j_{n}^{\prime}}$ be two different codewords in $\mathscr{C}_{D}$. Then we have

$$
\begin{aligned}
& \left|C_{j_{1}, j_{2}, \ldots, j_{n}} C_{j_{1}^{\prime}, j_{2}^{\prime}, \ldots, j_{n}^{\prime}}^{H}\right|=\frac{1}{K} \mid \sum_{i=1}^{K} \chi_{1, j_{1}}\left(g_{i, 1}\right) \chi_{2, j_{2}}\left(g_{i, 2}\right) \\
& \ldots \chi_{n, j_{n}}\left(g_{i, n}\right) \cdot \bar{\chi}_{1, j_{1}^{\prime}}\left(g_{i, 1}\right) \bar{\chi}_{2, j_{2}^{\prime}}\left(g_{i, 2}\right) \ldots \bar{\chi}_{n, j_{n}^{\prime}}\left(g_{i, n}\right) \mid \\
& =\frac{1}{K} \mid \sum_{i=1}^{K}\left(\chi_{1, j_{1}} \bar{\chi}_{1, j_{1}^{\prime}}\right)\left(g_{i, 1}\right)\left(\chi_{2, j_{2}} \bar{\chi}_{2, j_{2}^{\prime}}\right)\left(g_{i, 2}\right) \\
& \cdots\left(\chi_{n, j_{n}} \bar{\chi}_{n, j_{n}^{\prime}}\right)\left(g_{i, n}\right)\left|=\frac{1}{K}\right|\left(\left(\chi_{1, j_{1}} \bar{\chi}_{1, j_{1}^{\prime}}\right)\right. \\
& \left.\otimes\left(\chi_{2, j_{2}} \bar{\chi}_{2, j_{2}^{\prime}}\right) \otimes \cdots \otimes\left(\chi_{n, j_{n}} \bar{\chi}_{n, j_{n}^{\prime}}\right)\right)(D) \mid .
\end{aligned}
$$

Since $C_{j_{1}, j_{2}, \ldots, j_{n}}$ and $C_{j_{1}^{\prime}, j_{2}^{\prime}, \ldots, j_{n}^{\prime}}$ are different, $\left(\chi_{1, j_{1}} \bar{\chi}_{1, j_{1}^{\prime}}\right) \otimes$ $\left(\chi_{2, j_{2}} \bar{\chi}_{2, j_{2}^{\prime}}\right) \otimes \cdots \otimes\left(\chi_{n, j_{n}} \bar{\chi}_{n, j_{n}^{\prime}}\right)$ is a nontrivial character of $\mathbb{F}_{q}$. So Lemma 7 yields

$$
\begin{aligned}
I_{\max }\left(\mathscr{C}_{D}\right) & \leq \frac{1}{K} \cdot \frac{\sqrt{q_{1}+f\left(q_{1}\right)} \cdots \sqrt{q_{n}+f\left(q_{n}\right)}}{2} \\
& =\frac{\sqrt{q_{1}+f\left(q_{1}\right)} \cdots \sqrt{q_{n}+f\left(q_{n}\right)}}{q_{1} \cdots q_{n}-1} .
\end{aligned}
$$

In this case, the Welch bound $I_{\text {Welch }}$ for the codebook $\mathscr{C}_{D}$ is

$$
I_{\text {Welch }}=\sqrt{\frac{N-K}{(N-1) K}}=\frac{\sqrt{q_{1} q_{2} \cdots q_{n}+1}}{q_{1} q_{2} \cdots q_{n}-1} .
$$

Hence,

$$
\begin{aligned}
& 1 \leq \frac{I_{\text {max }}\left(\mathscr{C}_{D}\right)}{I_{\text {Welch }}} \leq \frac{\sqrt{q_{1}+f\left(q_{1}\right)} \cdots \sqrt{q_{n}+f\left(q_{n}\right)}}{\sqrt{q_{1} q_{2} \cdots q_{n}+1}} \\
& \quad \longrightarrow 1
\end{aligned}
$$

if $q_{i} \longrightarrow+\infty$ for any $1 \leq i \leq n$.

Corollary 9. With the above notations, we assume that all $\mathbb{F}_{q_{i}}$ are the same; that is, $q_{i}=q, i=1,2, \ldots, n$. In this case, the codebook $\mathscr{C}_{D}$ is a $\left(q^{n},\left(q^{n}-1\right) / 2\right)$ codebook satisfying

$$
I_{\max }\left(\mathscr{C}_{D}\right) \leq \frac{(\sqrt{q+f(q)})^{n}}{q^{n}-1} .
$$

In this case, $I_{\text {Welch }}=\sqrt{q^{n}+1} /\left(q^{n}-1\right)$. So

$$
1 \leq \frac{I_{\max }\left(\mathscr{C}_{D}\right)}{I_{\text {Welch }}} \leq \frac{(\sqrt{q+f(q)})^{n}}{\sqrt{q^{n}+1}} \rightarrow 1
$$

if $q \longrightarrow+\infty$.

For the case $D_{i}=C_{0}^{\left(2, q_{i}\right)} \subset \mathbb{F}_{q_{i}}, q_{i} \equiv 1(\bmod 4)$, we have the following lemma.

Lemma 10. For any character $\chi_{1, j_{1}} \otimes \chi_{2, j_{2}} \otimes \cdots \otimes \chi_{n, j_{n}}$ of $\mathbb{F}_{q}, j_{i}=0,1, \ldots, q_{i}-1, q_{i} \equiv 1(\bmod 4), i=1,2, \ldots, n$, if $\left(j_{1}, j_{2}, \ldots, j_{n}\right)=(0,0, \ldots, 0)$, we have

$$
\left|\left(\chi_{1, j_{1}} \otimes \chi_{2, j_{2}} \otimes \cdots \otimes \chi_{n, j_{n}}\right)(D)\right|=K .
$$

If $\left(j_{1}, j_{2}, \ldots, j_{n}\right) \neq(0,0, \ldots, 0)$,

$$
\begin{gathered}
\max \left|\left(\chi_{1, j_{1}} \otimes \chi_{2, j_{2}} \otimes \cdots \otimes \chi_{n, j_{n}}\right)(D)\right| \\
=\frac{\left(\sqrt{q_{1}}+1\right) \cdots\left(\sqrt{q_{n}}+1\right)}{2} .
\end{gathered}
$$

Proof. The proof is very similar to the proof of Lemma 7. We omit it here. 
Theorem 11. Let $\mathscr{C}_{D}$ be a codebook defined by (25) in the case of $D_{i}=C_{0}^{\left(2, q_{i}\right)} \subset \mathbb{F}_{q_{i}}, q_{i} \equiv 1(\bmod 4)$. Then $\mathscr{C}_{D}$ is an $(N, K)=$ $\left(q_{1} q_{2} \ldots q_{n},\left(q_{1} q_{2} \ldots q_{n}-1\right) / 2\right)$ codebook with

$$
I_{\text {max }}\left(\mathscr{C}_{D}\right)=\frac{\left(\sqrt{q_{1}}+1\right) \cdots\left(\sqrt{q_{n}}+1\right)}{q_{1} \cdots q_{n}-1}
$$

and $I_{\max }\left(\mathscr{C}_{D}\right)$ can reach the Welch bound if $q_{i} \longrightarrow \infty$ for any $1 \leq i \leq n$.

Proof. The proof is very similar to the proof of Theorem 8 . We omit it here.

Remark 12. The result of Theorem 11 is similar to that of [14]. The difference between our result and that of [14] is that Hu's method is based on the product of difference sets in finite Abelian group $G_{i}$ of order $N_{i}$, where $N_{i} \equiv 3(\bmod 4)$, whereas our method is based on the product of almost difference sets in finite field $\mathbb{F}_{q_{i}}$, where $q_{i} \equiv 1(\bmod 4)$.

Corollary 13. With the above notations, we assume that all $\mathbb{F}_{q_{i}}$ are the same; that is, $q_{i}=q \equiv 1(\bmod 4), i=1,2, \ldots, n$. In this case, the codebook $\mathscr{C}_{D}$ is a $\left(q^{n},\left(q^{n}-1\right) / 2\right)$ codebook satisfying

$$
I_{\max }\left(\mathscr{C}_{D}\right)=\frac{(\sqrt{q}+1)^{n}}{q^{n}-1} .
$$

In this case, $I_{\text {Welch }}=\sqrt{q^{n}+1} /\left(q^{n}-1\right)$. So

$$
1 \leq \frac{I_{\max }\left(\mathscr{C}_{D}\right)}{I_{\text {Welch }}}=\frac{(\sqrt{q}+1)^{n}}{\sqrt{q^{n}+1}} \longrightarrow 1
$$

if $q \longrightarrow+\infty$.

Remark 14. If $n=1$, our codebooks (25) in the case of $D_{i}=C_{0}^{\left(8, q_{i}\right)} \cup C_{1}^{\left(8, q_{i}\right)} \cup C_{2}^{\left(8, q_{i}\right)} \cup C_{5}^{\left(8, q_{i}\right)} \subset \mathbb{F}_{q_{i}}, q_{i} \equiv 9(\bmod 16)$, turn into the asymptotically optimal codebooks in [10], and our condition is milder, since [10] requires that $|x-a|$ and $|y|$ are bounded, whereas we require $\operatorname{limit}_{q->\infty}|x-a| / q=0$ and $\operatorname{limit}_{q->\infty}|y| / q=0$. If $n=1$, in the case of $D_{i}=$ $C_{0}^{\left(2, q_{i}\right)} \subset \mathbb{F}_{q_{i}}, q_{i} \equiv 1(\bmod 4)$, codebooks $(25)$ turn into the asymptotically optimal codebooks in [19].

Remark 15. By Remark 4 and Lemma 7, Theorem 8 still holds if we use $D_{i}=C_{l}^{\left(8, q_{i}\right)} \cup C_{1+l}^{\left(8, q_{i}\right)} \cup C_{2+l}^{\left(8, q_{i}\right)} \cup C_{5+l}^{\left(8, q_{i}\right)}$ instead of $D_{i}=C_{0}^{\left(8, q_{i}\right)} \cup C_{1}^{\left(8, q_{i}\right)} \cup C_{2}^{\left(8, q_{i}\right)} \cup C_{5}^{\left(8, q_{i}\right)}, l=1, \ldots, 7$. Similarly, by Remark 5 and Lemma 10, Theorem 11 still holds if we use $D_{i}=C_{1}^{\left(2, q_{i}\right)}$ instead of $D_{i}=C_{0}^{\left(2, q_{i}\right)}$.

\section{Conclusions}

In this paper, we construct a new class of asymptotically optimal codebooks based on the product of two classes of almost difference sets in finite fields. Future research is required to investigate whether this strategy works for any almost difference set of $(q,(q-1) / 2, \lambda, t)$ type. It is also interesting to modify the strategy such that we can start with MWBE codebooks and then derive new MWBE codebooks via a similar direct product. Another interesting problem is whether any complement of asymptotically codebooks similar to the Naimark complement of MWBE codebooks exists.

\section{Data Availability}

The data used to support the findings of this study are available from the corresponding author upon request.

\section{Conflicts of Interest}

The authors declare that there are no conflicts of interest regarding the publication of this paper.

\section{Acknowledgments}

This work is supported by the National Natural Science Foundation of China (nos. 61502217, 11404051, and 61702244).

\section{References}

[1] L. R. Welch, "Lower bounds on the maximum cross correlation of signals," IEEE Transactions on Information Theory, vol. 20, no. 3, pp. 397-399, 1974.

[2] M. Fickus and D. G. Mixon, "Tables of the existence of equiangular tight frames," https://arxiv.org/abs/1504.00253.

[3] D. V. Sarwate, "Meeting the welch bound with equality," in Sequences and their Applications, C. Ding, T. Helleseth, and H. Niederreiter, Eds., pp. 79-102, Springer, London, UK, 1999.

[4] J. M. Renes, "Equiangular tight frames from Paley tournaments," Linear Algebra and its Applications, vol. 426, no. 2-3, pp. 497-501, 2007.

[5] C. Ding and T. Feng, "A generic construction of complex codebooks meeting the Welch bound," Institute of Electrical and Electronics Engineers Transactions on Information Theory, vol. 53, no. 11, pp. 4245-4250, 2007.

[6] M. Fickus, D. G. Mixon, and J. C. Tremain, "Steiner equiangular tight frames," Linear Algebra and its Applications, vol. 436, no. 5, pp. 1014-1027, 2012.

[7] M. A. Sustik, J. A. Tropp, I. S. Dhillon, and R. W. Heath Jr., "On the existence of equiangular tight frames," Linear Algebra and its Applications, vol. 426, no. 2-3, pp. 619-635, 2007.

[8] C. Ding and T. Feng, "Codebooks from almost difference sets," Designs, Codes and Cryptography. An International Journal, vol. 46, no. 1, pp. 113-126, 2008.

[9] A. Zhang and K. Feng, "Construction of cyclotomic codebooks nearly meeting the Welch bound," Designs, Codes and Cryptography. An International Journal, vol. 63, no. 2, pp. 209-224, 2012.

[10] Z. Aixian and K. Feng, "Construction of a class of codebooks nearly meeting the Welch bound," Scientia Sinica Informationis, vol. 45, no. 12, p. 1632, 2015.

[11] N. Y. Yu, "A construction of codebooks associated with binary sequences," Institute of Electrical and Electronics Engineers Transactions on Information Theory, vol. 58, no. 8, pp. 55225533, 2012.

[12] Z. Heng, "Nearly optimal codebooks based on generalized Jacobi sums," Discrete Applied Mathematics: The Journal of Combinatorial Algorithms, Informatics and Computational Sciences, vol. 250, pp. 227-240, 2018. 
[13] G. Luo and X. Cao, "Two constructions of asymptotically optimal codebooks via the hyper Eisenstein sum," Institute of Electrical and Electronics Engineers Transactions on Information Theory, vol. 64, no. 10, pp. 6498-6505, 2018.

[14] H. Hu and J. Wu, "New constructions of codebooks nearly meeting the Welch bound with equality," Institute of Electrical and Electronics Engineers Transactions on Information Theory, vol. 60, no. 2, pp. 1348-1355, 2014.

[15] C. Ding, Codes from Difference Sets, World Scientific Publishing Co., 2014.

[16] E. Lehmer, "On the number of solutions of $u^{k}+D w^{2}(\bmod p)$," Pacific Journal of Mathematics, vol. 5, no. 1, pp. 103-118, 1955.

[17] C. Ding, A. Pott, and Q. Wang, "Constructions of almost difference sets from finite fields," Designs, Codes and Cryptography. An International Journal, vol. 72, no. 3, pp. 581-592, 2014.

[18] R. Lidl and H. Niederreiter, Finite Fields, vol. 20, Cambridge University Press, Cambridge, UK, 1997.

[19] C. Ding, "Complex codebooks from combinatorial designs," Institute of Electrical and Electronics Engineers Transactions on Information Theory, vol. 52, no. 9, pp. 4229-4235, 2006. 


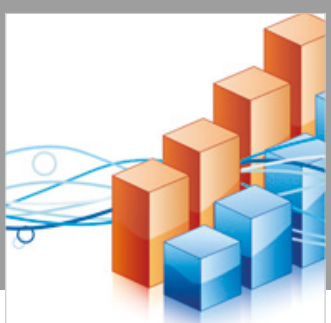

Advances in

Operations Research

\section{-n-m}
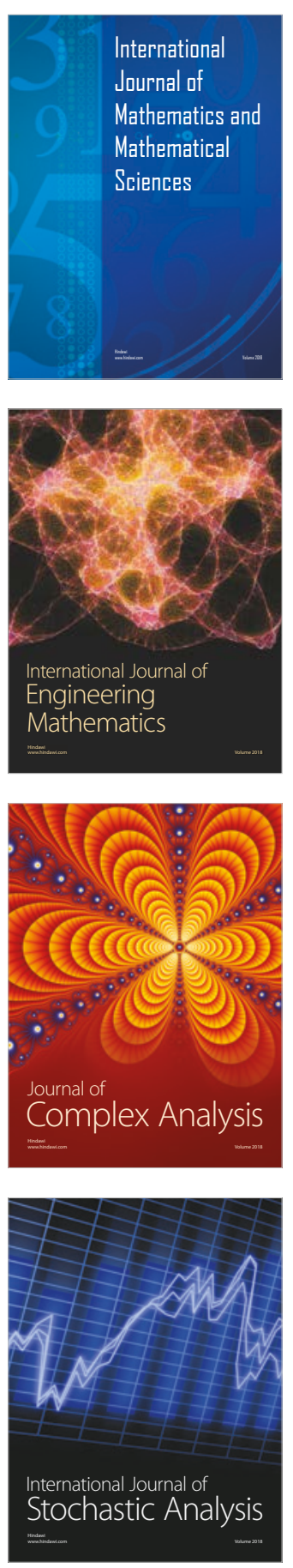
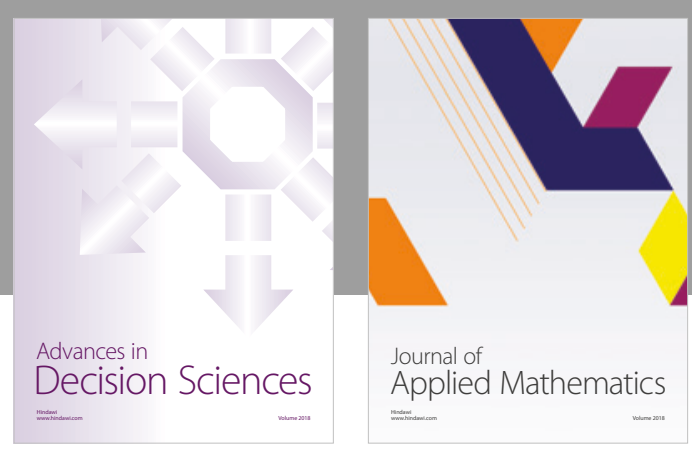

Journal of

Applied Mathematics
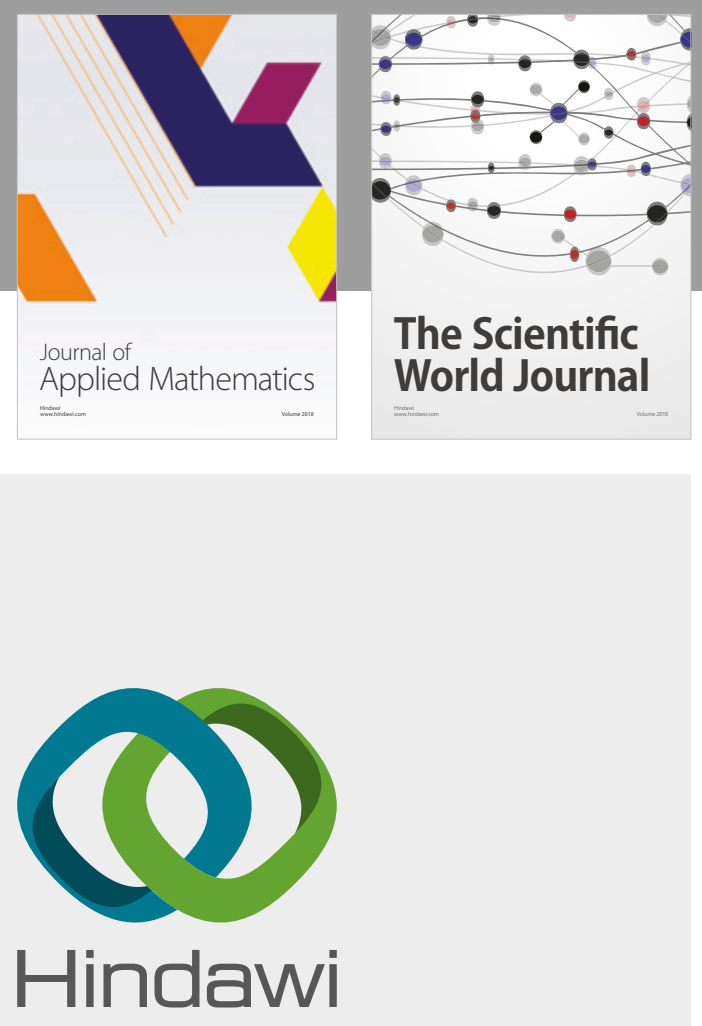

Submit your manuscripts at

www.hindawi.com

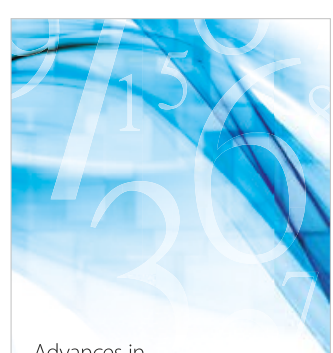

Advances in
Numerical Analysis
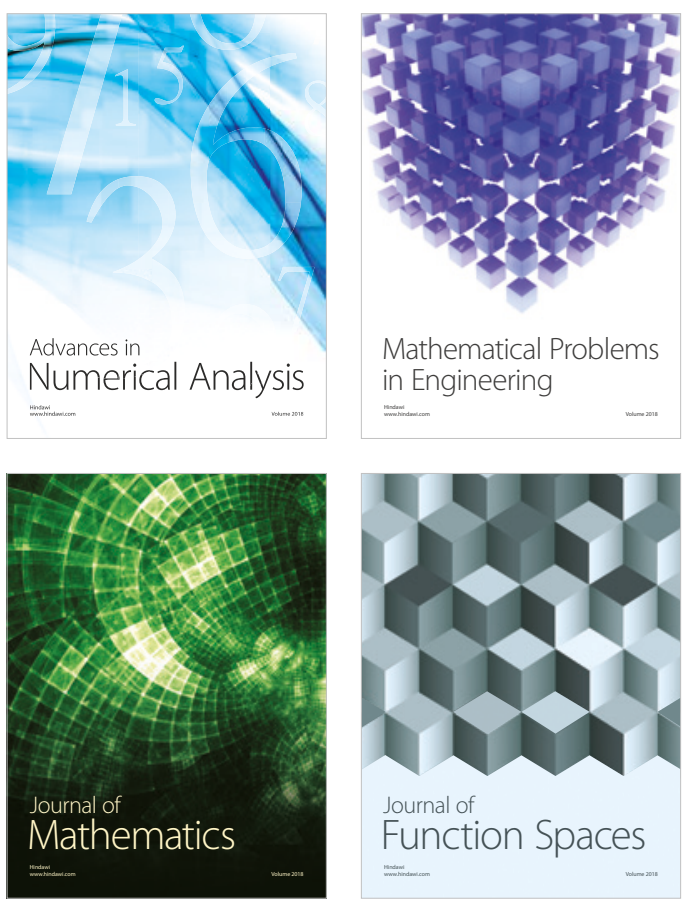

Mathematical Problems in Engineering

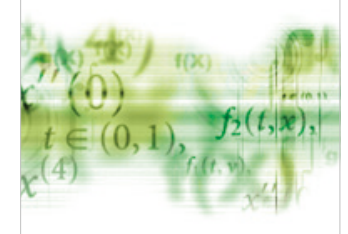

International Journal of

Differential Equations

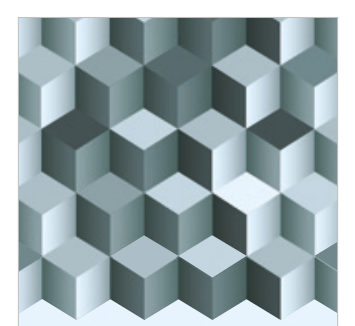

Journal of

Function Spaces

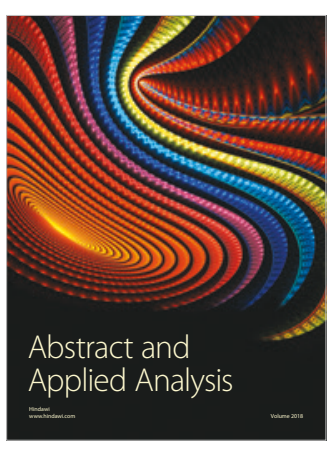

The Scientific

World Journal

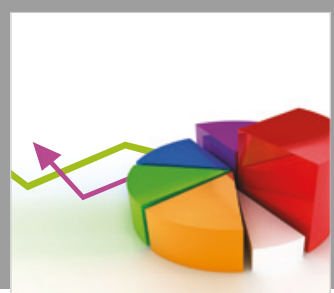

Journal of

Probability and Statistics
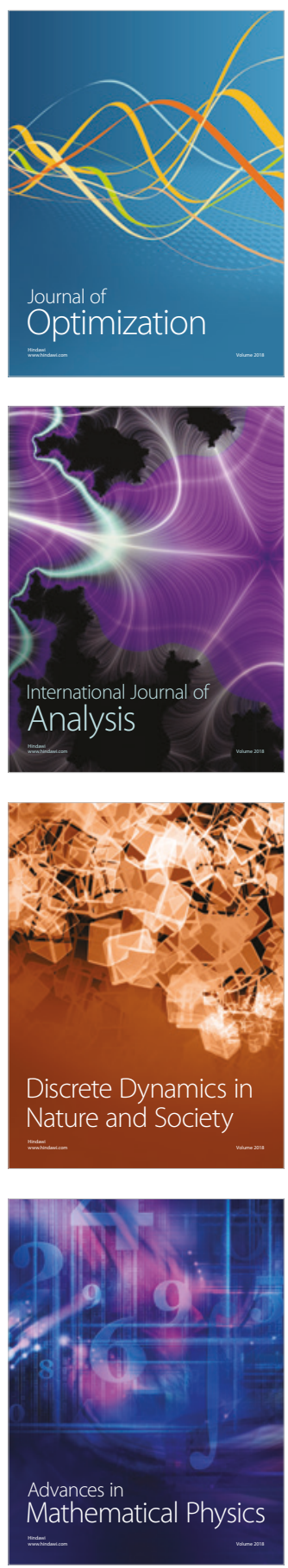